\title{
Interactive comment on "Model-driven
} optimization of coastal sea observatories through data assimilation in a finite element hydrodynamic model (SHYFEM v.7_5_65)" by Christian Ferrarin \section{et al.}

Christian Ferrarin et al.

c.ferrarin@ismar.cnr.it

Received and published: 22 June 2020

Dear Reviewer,

We would like to thank you very much for your tireless efforts in reviewing the manuscript and for your valuables comments, which will certainly improve our work.

The original Reviewer's comments and suggestions are shown in regular typeface, while our responses are shown in italics. 
R1.1 General comments: "Model-driven optimization of coastal sea observatories through data assimilation in a finite element hydrodynamic model (SHYFEM v.7_5_65)" is a well written manuscript concerned with using data assimilation to improve coastal modelling capabilities and optimise monitoring networks. The article contains a fair comparison of data interpolation (DI) and data assimilation (DA) methods, along with a further comparison of two different DA approaches. The Lagoon of Venice application involves a complex spatial domain with sensitive coastal dynamics. The objective of the numerical experimentation is clearly stated. Results are clearly presented in a number of attractive figures. In the conclusion, the objective is fulfilled and recommendations are made for modifying the monitoring network.

Response: We appreciate the comments and we improved the manuscript following all reviewer's suggestions.

R1.2 I particularly liked the introduction to DI and DA philosophy given in the paragraph starting on line 37.

Response: We thank the reviewer for this positive comment.

R1.3 Equations (6)-(7) could perhaps be introduced in a better way. There is a lot of notation all at once, some of which is not referred to in the text. For example, it would be good to elaborate on what is meant by the superscript "a" for "analysis" (although this does become clear at the end of Section 2).

Response: We concur with the reviewer that some of the mathematical passages were poorly explained. In the revised manuscript, we improved the explanation of the mentioned equations by adding more details on the different terms.

R1.4 In the paragraph beginning on line 201, it would be beneficial to clarify how unforced boundary conditions are represented within the shallow water model. Forced boundaries are mentioned, but the implementation of unforced boundary conditions (for example in urban areas) is unclear. Are free-slip conditions used?

Printer-friendly version

Discussion paper 
Response: Unforced boundaries are solid boundaries that are implemented in the model with a free slip condition. The only condition that is enforced on these boundaries are the no-flux condition through these boundaries. No-slip conditions can also be implemented by the model, however, the resolution of the numerical grid is much too coarse for these kind of condition.

In the revised manuscript, we have inserted the following sentence: "At the boundaries, either water levels are prescribed at the open boundaries or the freeslip condition is implemented at solid (closed) boundaries".

R1.5 The statement on lines 274-275 claims that "results improved at all stations". However, there appears to be one exception at station 12, where the control simulation RMSE is 4.5 but the DA-EnSRF RMSE is 4.7. This should at least be mentioned and a sentence suggesting a reason for the anomaly would be beneficial.

Response: We thank the reviewer for highlighting this anomaly which was due to a typesetting error in the ${ }^{2} T_{E} X$ file. The correct value of 3.7 is now reported in Table 1.

R1.6 There are a small number of typos and grammatical errors in the current manuscript.

Response: We corrected all reported typos and grammatical errors.

Interactive comment on Geosci. Model Dev. Discuss., https://doi.org/10.5194/gmd-2020-61, 2020.

Printer-friendly version

Discussion paper 